\title{
Metrization, paracompactness, and real-valued functions
}

J. A. Guthrie (El Paso, Tex.) and M. Henry (Morgantown, W. Va.)

Abstract. A $T_{0}$ space $X$ is metrizable if and only if it has the weak topology induced by countably many collections $\mathcal{F}_{n}=\left\{f_{n \alpha} \mid \alpha \epsilon A_{n}\right\}$ of real-valued functions on $X$, each having the property that for $B_{n} \subset A_{n}$, inf $\left\{f_{n \alpha} \mid \alpha \in B_{n}\right\}$ and $\sup \left\{f_{n \alpha} \mid \alpha \in B_{n}\right\}$ are continuous. A similar result yields a characterization of paracompactness.

The main results of this paper were announced in [1].

In 1925, P. Urysohn proved the following theorem which now bears his name. A separable space $X$ is metrizable iff $X$ is $T_{3}$ and second countable. Of course the Urysohn Metrization Theorem characterizes only those spaces which are separable metrizable, leaving unanswered the question of how to characterize arbitrary metrizable spaces. As is well-known, this question was answered in 1950 and 1951 by Nagata, Smirnov, and Bing who obtained more general results which do not assume separability.

The usual direct proof of the Urysohn Metrization Theorem utilizes the following well-known result.

THEOREM 1. A $T_{0}$ space $X$ is separable metrizable if and only if $X$ has the weak topology induced by countably many real-valued functions.

By definition, the weak topology induced by a collection $\left\{f_{\alpha}: \alpha \in A\right\}$ of realvalued functions is the topology for which $\left\{f_{\alpha}^{-1}(U): \alpha \in A, U\right.$ open in $\left.R\right\}$ is a subbase.

There are also other forms that Theorem 1 may assume. In some versions the functions may separate points (replacing the $T_{0}$ condition), while in others the functions may separate points from closed sets (a stronger assumption than requiring the weak topology condition). But in any event, separability must be assumed.

Just as the Urysohn Metrization Theorem is a corollary to the Nagata-Smirnov Theorem, it is the purpose of this paper to show that Theorem 1 is, itself, a special case of a more general result (Theorem 2) that does not rely on separability. Furthermore, it will be shown that this result can be applied to prove the NagataSmirnov Theorem in virtually the same manner that Theorem 1 is used in proving 
Urysohn's Metrization Theorem, thus indicating that Theorem 2 is indeed the desired generalization. Finally, in proving Theorem 2, a characterization of paracompact spaces (Theorem 9) will be established which improves the characterization involving locally finite partitions of unity.

Let us say that a collection $\left\{f_{\alpha}: \alpha \in A\right\}$ of real-valued functions defined on a topological space is relatively complete if the functions $\inf \left\{f_{\beta}: \beta \in B\right\}$ and $\sup \left\{f_{\beta}: \beta \in B\right\}$ are continuous for each $B \subset A$, and let us say that a collection $\mathscr{F}=\bigcup_{n} \mathscr{F}_{n}$ of real-valued functions is $\sigma$-relatively complete if each $\mathscr{F}_{n}$ is relatively complete. We now prove the main theorem.

THEOREM 2. A T $T_{0}$ space $X$ is metrizable if and only if $X$ has the weak topology induced by a $\sigma$-relatively complete collection.

Proof. Necessity follows from Theorem 5, which is due to Nagata. To prove the sufficiency, let $\mathscr{F}=\bigcup \mathscr{F}_{n}$ be a $\sigma$-relatively complete collection where $\mathscr{F}_{n}$ $=\left\{f_{n \alpha}: \alpha \in A_{n}\right\}$. We may assume that $\mathscr{F}_{n} \subset \mathscr{F}_{n+1}$. Since $X$ is $T_{0}$ and has the weak topology, $X$ must be Hausdorff. By Theorem $9, X$ is paracompact, and we will be done if we can show that $X$ is also developable.

For rationals $a, b, s, t$, let

$$
\begin{aligned}
& A(n, s, t, x)=\left\{\alpha \in A_{n}: f_{n \alpha}(x)<\frac{1}{2}(s+t)\right\}, \\
& B(n, a, b, x)=\left\{\alpha \in A_{n}: f_{n \alpha}(x)>\frac{1}{2}(a+b)\right\}
\end{aligned}
$$

and whenever $a<b<s<t$, let

$$
U(n, a, b, s, t, x)=K \cap L \cap M \cap N
$$

where

$$
\begin{aligned}
& K=\left\{y: \sup _{\alpha} f_{n \alpha}(y)<t, \alpha \in A(n, s, t, x)\right\}, \\
& L=\left\{y: \inf _{\alpha} f_{n \alpha}(y)>s, \alpha \notin A(n, s, t, x)\right\}, \\
& M=\left\{y: \inf _{\alpha} f_{n \alpha}(y)>a, \alpha \in B(n, a, b, x)\right\}, \\
& N=\left\{y: \sup _{\alpha} f_{n \alpha}(y)<b, \alpha \notin B(n, a, b, x)\right\} .
\end{aligned}
$$

Each $U(n, a, b, s, t, x)$ is a nbd of $x$ and there are countably many collections $\mathscr{U}(n, a, b, s, t)=\{U(n, a, b, s, t, x): x \in X\}$, each obviously being a cover of $X$. For each finite set $F$ of quintuples $\left(n_{i}, a_{i}, b_{i}, s_{t}, t_{i}\right)$ of rationals, choose the largest $n$ among the $n_{i}$ 's, and let $\varkappa_{F}$ be the set of all finite intersections of members of $\mathscr{U}\left(n, a_{i}, b_{i}, s_{i}, t_{i}\right)$ where an intersection consists of one member from each $\mathscr{U}$. Then the collection of all such $\mathscr{U}_{F}$ 's is countable, and we will show that $\left\{\mathscr{U}_{F}\right\}$ forms a development for $X$.

To this end, let $p \in V$ where $V$ is any nbd. There exist integers $n, k$ and rationals $a_{i}, t_{i}(i=1, \ldots, k)$ for which $p \in \bigcap_{i} f_{n \alpha_{i}}^{-1}\left(\left(a_{i}, t_{i}\right)\right) \subset V$. If we choose rationals $b_{i}, s_{i}$ such that $a_{i}<b_{i}<f_{n \alpha_{i}}(p)<s_{i}<t_{i}$, then the corresponding collection $\mathscr{U}_{F}$ defined in the preceding paragraph will satisfy the condition $\operatorname{St}\left(p, \mathscr{U}_{F}\right) \subset V$. To verify this, consider an arbitrary set $W \in \mathscr{U}_{F}$. We know that $W=\bigcap_{i} U\left(n, a_{i}, b_{i}, s_{i}, t_{i}, x_{i}\right)$, and we observe that if $\alpha_{i} \notin A\left(n, s_{i}, t_{i}, x_{i}\right)$ for some $i=1, \ldots, k$, then $p \notin W$. The reason for this is that $U\left(n, a_{i}, b_{i}, s_{i}, t_{i}, x_{i}\right) \subset L_{i}$ and $p \notin L_{i}$ since $f_{n \alpha_{i}}(p)<s_{i}$. Similarly, if $\alpha_{i} \notin B\left(n, a_{i}, b_{i}, x_{i}\right)$ then $p \notin W$. Thus, if $p \in W$, then $\alpha_{i} \in A\left(n, s_{i}, t_{i}, x_{i}\right)$, $B\left(n, a_{i}, b_{i}, x_{i}\right)$ for each $i=1, \ldots, k$, and this implies that

$$
\begin{aligned}
W & \subset \bigcap_{i}\left[K_{i} \cap M_{i}\right] \\
& \subset \bigcap_{i}\left[f_{n \alpha_{i}}^{-1}\left(\left(-\infty, t_{i}\right)\right) \cap f_{n \alpha_{i}}^{-1}\left(\left(a_{i}, \infty\right)\right)\right] \\
& =\bigcap_{i} f_{n \alpha_{i}}^{-1}\left(\left(a_{i}, t_{i}\right)\right) \\
& \subset V .
\end{aligned}
$$

Hence, $\operatorname{St}\left(p, \mathscr{U}_{F}\right) \subset V$, and the proof is complete.

Theorem 3 (Nagata-Smirnov). $A T_{3}$ space $X$ is metrizable if and only if $X$ has a $\sigma$-locally finite base.

Proof. Let $U \mathscr{B}_{n}$ be a $\sigma$-locally finite base. For each $U \in \mathscr{B}_{n}$, let

$$
V=U\left\{W \in \mathscr{B}_{m}: \bar{W} \subset U\right\} \text {. }
$$

Then $\bar{V} \subset U$ and hence there is a continuous $f_{U V}: X \rightarrow[0,1]$ such that $f_{U V}(\bar{V})=1$ and $f_{U V}(X-U)=0$. If we let $\mathscr{F}_{m n}=\left\{f_{U V}: \bar{V} \subset U\right\}$, then each $\mathscr{F}_{m n}$ will be relatively complete because of the local finiteness of $\mathscr{B}_{n}$, and it is easy to show that $X$ has the weak topology induced by $\bigcup \mathscr{F}_{m n}$.

It should be pointed out that the collection of functions $\bigcup \mathscr{F}_{m n}$ constructed in the proof of the Nagata-Smirnov Theorem is indeed a special case of the ones to which Theorem 2 applies. By definition, the support of a function $f: X \rightarrow R$ is the closed set $\{y: f(y) \neq 0\}$. Thus, each collection $\mathscr{F}_{m n}$ was trivially seen to be relatively complete because the supports of the members of $\mathscr{F}_{m n}$ formed a locally finite collection. The following example shows that a collection $\mathscr{F}$ of real-valued functions need not have locally finite supports in order to be relatively complete.

EXAMPLE 4. Let $R$ be the real line with the usual topology, and define for each $y \in R$

$$
f_{y}(x)=\left\{\begin{array}{lll}
1 & \text { if } & x \leqslant y-1 \\
y-x & \text { if } & y-1<x<y+1 \\
-1 & \text { if } & y+1 \leqslant x .
\end{array}\right.
$$

Clearly each $f_{y}$ is continuous and $\mathscr{F}=\left\{f_{y}: y \in R\right\}$ induces the usual topology on $R$.

Now for $A \subset R$, let $F_{A}(x)=\inf \left\{f^{\prime}(x): y \in A\right\}$ and let $G_{A}(x)=\sup \{f(x): y \in A\}$. If $\inf \{y: y \in A\}$ does not exist, then $F_{A}(x)=-1$ for each $x \in X$, while if inf $\{y: y \in A\}$ $=m$ exists, then $F_{A}(x)=f_{m}(x)$ for each $x \in X$. Thus $F_{A}(x)$ is continuous for each $A \subset R$. Similarly, $G_{A}(x)=1$ or $G_{A}(x)=f_{M}(x)$ where $M=\sup \{y: y \in A\}$. This shows that $\mathscr{F}$ is relatively complete. 
Finally, note that $\mathscr{F}$ cannot be decomposed into countably many collections each having point-finite support, because the support of each $f_{y} \in \mathscr{F}$ is $R$.

We remark at this point that Theorem 2 is not the first characterization of metrizability in terms of the existence of real-valued functions, nor is the concept of a collection of real-valued functions having continuous infs and sups a new one. In 1957, J. Nagata proved the following [4, Theorem 5]:

THEOREM 5. A $T_{1}$ space $X$ is metrizable if and only if there exists a family $\left\{f_{\alpha}: \alpha \in A\right\}$ of real-valued functions on $X$ such that

(1) $\inf \left\{f_{\beta}: \beta \in B\right\}$ and $\sup \left\{f_{\beta}: \beta \in B\right\}$ are continuous for each $B \subset A$.

(2) For any $n b d U(x)$ of any point $x \in X$ there exists $\alpha \in A$ and a real number : such that $f_{\alpha}(x)<\varepsilon, f_{\alpha}((X-U(x)) \geqslant \varepsilon$.

The relationship between Theorem 5 and Theorem 2 is easily seen upon observing that condition (2) of Theorem 5 is equivalent to the following condition $\left(2^{\prime}\right)$. for $X$.

$\left.2^{\prime}\right)$ The collection $\left\{f_{\alpha}^{-1}((-\infty, \varepsilon)): \alpha \in A, \varepsilon\right.$ a real number $\}$ forms a base

Thus, Theorem 2 is an improvement of Theorem 5.

The next example shows that one cannot improve Theorem 2 by requiring only infs or only sups to be continuous.

EXAMPLE 6. Let $S$ be the Sorgenfrey line; that is, the real numbers with the topology having as a base all intervals of the form $[a, b)$. Define for each $y \in S$

$$
f_{y}(x)= \begin{cases}x-y & \text { if } y \leqslant x<y+1 \\ 1 & \text { otherwise } .\end{cases}
$$

Clearly $f_{y}$ is continuous and $\mathscr{F}=\left\{f_{y}: y \in S\right\}$ not only induces the topology of $S$, but has property (2) of Theorem 5 .

Now for $A \subset S$ let $D(A)$ be the Euclidean diameter of $A$, and let $F_{A}(x)$ $=\sup \left\{f_{y}(x): y \in A\right\}$. If $D(A)>1, F_{A}(x)=1$ for every $x \in S$. If $D(A)<1$, let $m$ $=\inf \{y: y \in A\}$ and let $M=\sup \{y: y \in A\}$. Then

$$
F_{A}(x)=\left\{\begin{array}{lll}
1 & \text { if } & x<M, \\
f_{m}(x) & \text { if } & x \geqslant M .
\end{array}\right.
$$

Thus $\mathscr{F}$ has continuous sups. It is well-known, however, that $S$ is not metrizable. Similarly, we may let $g_{y}(x)=-f_{y}(x)$. Then $\mathscr{G}=\left\{g_{y}: y \in S\right\}$ has continuous infs and induces the topology of $S$.

Finally, let us shift our attention to the characterization of paracompactness (Theorem 9) used in the proof of Theorem 2. We recall first the following definitions and theorems.

By a partition of unity on a space $X$, we mean a collection of continuous functions from $X$ into $[0,1]$ such that $\sum\{f(x): f \in \mathscr{F}\}=1$ for each $x \in X$. A partition of unity $\mathscr{F}$ is locally finite if the supports of the members of $\mathscr{F}$ form a locally finite closed cover of $X$, and $\mathscr{F}$ is subordinated to a cover $\mathscr{U}$ of $X$ if the supports
refine $\mathscr{U}$.
Theorem 7 (Michael [2, Proposition 2]). The following properties of a $T_{1}$ space $X$ are equivalent:

(1) $X$ is paracompact,

(2) Every open cover of $X$ has a locally finite partition of unity subordinated to it.

A cover $\mathscr{V}$ is cushioned in a cover $\mathscr{U}$ if one can assign to each $V \in \mathscr{V}$ a $U_{V} \in \mathscr{U}$ such that for every $\left.\mathscr{V}^{\prime} \subset \mathscr{V}, \overline{U\left\{V: V \in \mathscr{V}^{\prime}\right.}\right\} \subset \bigcup\left\{U_{V}: V \in \mathscr{V}^{\prime}\right\} . \mathscr{V}$ is a $\sigma$-cushioned refinement of $\mathscr{U}$ if $\mathscr{V}=\bigcup\left\{\mathscr{V}_{i}: i=1,2, \ldots\right\}$ with each $\mathscr{V}_{i}$ cushioned in $\mathscr{U}$ and $\mathscr{V}$ refines $\mathscr{U}$.

Theorem 8 (Michael [3, Theorem 1.2]). A $T_{1}$ space $X$ is paracompact if and only if every open cover of $X$ has an open $\sigma$-cushioned refinement.

Trreorem 9. A $T_{1}$-space $X$ is paracompact if and only if for each open cover $\mathscr{W}$, there is a $\sigma$-relatively complete collection $\bigcup_{n}\left\{f_{n \alpha}: \alpha \in A_{n}\right\}$ and a refinement of $\mathscr{W}$ consisting of sets of the form $\bigcap_{i=1}^{m} f_{n_{i} \alpha_{i}}^{-1}\left(U_{i}\right)$ where $U_{i}$ is open in $R$.

Proof. Necessity. By Theorem 7, $X$ has a locally finite partition of unity $\mathscr{F}$ subordinated to $\mathscr{W}$, and it is easy to verify that $\mathscr{F}$ is relatively complete and has the desired refinement property.

Sufficiency. Let $\mathscr{F}=\bigcup_{n}\left\{f_{n \alpha}: \alpha \in A_{n}\right\}$ be a $\sigma$-relatively complete collection having the given refinement property. We may assume that $\mathscr{F}_{n} \subset \mathscr{F}_{n+1}$. We will show that $X$ is paracompact by constructing an open $\sigma$-cushioned refinement of $\mathscr{W}$.

For each positive integer $n$, and each pair of rationals $b, s$ with $b<s$, let $U(n, \alpha, b, s)=f_{n \alpha}^{-1}((b, s))$ and let $\mathscr{U}(n, b, s)=\left\{U(n, \alpha, b, s): \alpha \in A_{n}\right\}$.

Each $\mathscr{U}(n, b, s)$ is cushioned in $\mathscr{U}(n, a, t)$ whenever $a<b<s<t$. The correspondence is $U(n, \alpha, b, s) \rightarrow U(n, \alpha, a, t)$ for each $\alpha \in A_{n}$. For suppose that there is an infinite subcollection $A \subset A_{n}$ for which $x \in \overline{\bigcup_{\alpha} U(n, \alpha, b, s)}-\bigcup_{\alpha} U(n, \alpha, a, t)$. Let $A^{\prime}=\left\{\alpha \in A: f_{n \alpha}(x)>t\right\}$ and let $A^{\prime \prime}=\left\{\alpha \in{ }^{\alpha}: f_{n \alpha}(x)<a\right\}$. By hypothesis, $f=\inf \left\{f_{n \alpha}: \alpha \in A^{\prime}\right\}$ is continuous, and therefore $f(x) \geqslant t$. This means that $f^{-1}\left(\left(\frac{1}{2}(s+t), \infty\right)\right)$ is a nbd of $x$ that misses $U(n, \alpha, b, s)$ for each $\alpha \in A^{\prime}$. Similarly, $F=\sup \left\{f_{n a}: \alpha \in A^{\prime \prime}\right\}$ is continuous, $F(x) \leqslant a$, and $F^{-1}\left(\left(-\infty, \frac{1}{2}(a+b)\right)\right)$ misses $U(n, \alpha, b, s)$ for each $\alpha \in A^{\prime \prime}$. Thus, the intersection of the two inverse image nbds is a nbd of $x$ that misses $\bigcup_{\alpha} U(n, \alpha, b, s)$, which is a contradiction.

We now proceed to construct countably many open cushioned families that refine $\mathscr{H}$. For rationals $a_{i}<b_{i}<s_{i}<t_{i}, i=1, \ldots, k$ we have that $\mathscr{U}=\bigcup_{i} \mathscr{U}\left(n_{i}, b_{i}, s_{i}\right)$ is cushioned in $\mathscr{F}=\bigcup_{i} \mathscr{U}\left(n_{i}, a_{i}, t_{i}\right)$ under the correspondence $\underset{i}{U}\left(n_{i}, \alpha, b_{i}, s_{i}\right)$ $\rightarrow U\left(n_{i}, \alpha, a_{i}, t_{i}\right)$ and there will be countably many such cushioned collections (in fact, each $\mathscr{O}$ is cushioned in infinitely many other $\mathscr{U}$ 's). Similarly, whenever $\mathscr{U}$ is cushioned in $\mathscr{V}$, the collection $\mathscr{U}^{*}$ of all finite intersections of members of $\mathscr{U}$ will be cushioned in the collection $\mathscr{V}^{*}$ of all finite intersections of members of $\mathscr{V}$ under the correspondence $\cap U\left(n_{i}, \alpha_{i}, b_{i}, s_{i}\right) \rightarrow \bigcap U\left(n_{i}, \alpha_{i}, a_{i}, t_{i}\right)$. Now for each such. 
cushioned pair with $\mathscr{U}^{*}$ cushioned in $\mathscr{V}^{*}$, let $\mathscr{U} \mathscr{V}=\left\{U \in \mathscr{U}^{*}: U \rightarrow V\right.$ where $V \in \mathscr{V}^{*}$ and $V \subset W$ for some $\left.W \in \mathscr{W}\right\}$. Then $\mathscr{U} \mathscr{V}$ is cushioned in $\mathscr{W}$ under the correspondence $U \rightarrow W$, there are countably many such collections, and we will be done if we can show that $U \mathscr{U} \mathscr{V}$ refines $\mathscr{W}$. This is easy to do, for given $x \in X$, there is a $W \in \mathscr{W}$ for which $x \in \bigcap_{i} U\left(n, \alpha_{i}, a_{i}, t_{i}\right) \subset W$. Choose rationals $b_{i}, s_{i}$ with $a_{i}<b_{i}<f_{n \alpha_{i}}(x)<s_{i}<t_{i}$ and note that the set $\bigcap_{i} U\left(n, \alpha_{i}, b_{i}, s_{i}\right)$ contains $x$ and is a member of some $\mathscr{U} \mathscr{V}$. Thus, $\mathscr{W}$ does indeed have an open $\sigma$-cushioned refinement, and the proof is complete.

Thus, Theorem 9 is more general than Theorem 7. Furthermore, Example 4 shows that there are relatively complete collections satisfying the hypotheses of Theorem 9 that are not locally finite partitions of unity.

\section{References}

[1] J. A. Guthrie and M. Henry, Metrization and paracompactness in terms of real functions, Bull. Amer. Math. Soc. 80 (1974), pp. 720.

[2] E. Michael, $A$ note on paracompact spaces, Proc. Amer. Math. Soc. 4 (1953), pp. 831-838.

[3] - Yet another note on paracompact spaces, Proc. Amer. Math. Soc. 10 (1959), pp. 309-314.

[4] J. Naga ta, A contribution to the theory of metrization, J. Inst. Polytech., Osaka City University, 8 (1957), pp. 185-192.

\section{UNIVERSTTY OF TEXAS AT EL PASO}

WEST VIRGINLA UNIVERSITY

Accepté par la Rédaction le 17. 2. 1975

\section{Locally well-behaved paracompacta in shape theory}

by

G. Kozlowski and J. Segal* (Seattle, Wash.)

Abstract. We generalize the classical notion of ANR to paracompacta in shape theory to obtain the notion of absolute neighborhood shape extensor (ANSE). Although the corresponding classical statement is false for compacta we have the theorem: Any $\mathrm{LC}^{n}$ paracompactum of dimension $\leqslant n$ is an ANSE. We also generalize the various notions of movability to arbitrary topological spaces.

THEOREM. Every $\mathrm{LC}^{n-1}$ paracompactum of dimension $\leqslant n$ is uniformly movable.

\section{Contents}

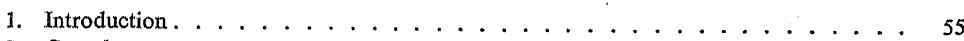

2. Complexes .............................. 56

3. Shape theory for topological spaces . . . . . . . .... 57

4. Movability and $n$-movability . . . . . . . . . . . . . . . . 60

5. ANSE's and ANSR's . . . . . . . . . . . . . . . . . . . . . . . $66_{65}$

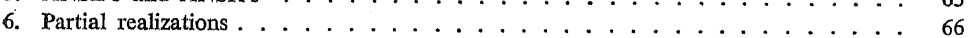

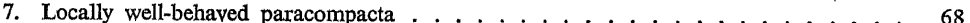

8. Summary ........................ 70

1. Introduction. In Section 3 we give a categorical description of shape theory for arbitrary topological spaces based on the concept of natural transformations of homotopy classes of maps into polyhedra. We show precisely in what sense this theory agrees with the Mardesić-Segal ANR-systems approach to shape theory on compacta.

K. Borsuk [2] introduced the notion of movability for metric compacta as a generalization of ANR's, and S. Mardešić and J. Segal [16] extended this property to compacta by means of ANR-systems: Movability appears to be the most interesting shape invariant discovered so far. It occurs as the hypothesis under which many classical theorems of algebraic topology generalize to shape theory, for example, [9] and [19]. In Section 4 we present a definition of movability for arbitrary

* The second named author was partially supported by NSF grant GP-34058. 